DISCLOSURE

In addition to income received from his primary employer, Dr David Steffens has received honoraria for speaking at educational activities sponsored by Forest Pharmaceuticals and Wyeth Pharmaceuticals. This work was partially supported by National Institute of Mental Health Grants P50 MH60451, R01 $\mathrm{MH} 54846$, and $\mathrm{K} 24 \mathrm{MH} 70027$.

Alexopoulos GS, Meyers BS, Young RC, Campbell S, Silbersweig D, Charlson M (1997). 'Vascular depression' hypothesis. Arch Gen Psychiatry 54: 915-922.

Pan CC, McQuoid DR, Taylor WD, Payne ME, AshleyKoch A, Steffens DC (2009). Association analysis of the COMT/MTHFR genes and geriatric depression: An MRI study of the putamen. Int $J$ Geriatr Psychiatry 24: 847-855.

Qiu A, Taylor WD, Zhao Z, MacFall JR, Miller MI, Key $\mathrm{CR}$ et al (2009). APOE related hippocampal shape alteration in geriatric depression. Neuroimage 44 620-626.

Steffens DC, Taylor WD, McQuoid DR, Krishnan KR (2008). Short/long heterozygotes at 5HTTLPR and white matter lesions in geriatric depression. Int J Geriatr Psychiatry 23: 244-248.

Taylor WD, Züchner S, McQuoid DR, Payne ME, MacFall JR, Steffens DC et al (2008). The brainderived neurotrophic factor VAL66MET polymorphism and cerebral white matter hyperintensities in late-life depression. Am J Geriatr Psychiatry 16: 263-271.

Taylor WD, Steffens DC, Ashley-Koch A, Payne ME, MacFall JR, Potocky C et al (2009). Angiotensin receptor gene polymorphisms and 2-year change in cerebral hyperintense lesion volume in men. Mol Psychiatry, March 10 (E-pub ahead of print).

Neuropsychopharmacology Reviews (2010) 35, 349-350; doi: I0.1038/npp.2009.135

\section{Neuronal signaling pathways: genetic insights into the pathophysiology of major mental illness}

Psychiatric genetics has turned a corner-increasingly robust findings can be placed in a neurobiological context, with practical implications for understanding disease pathogenesis and developing of therapeutics. A milestone in this effort was the discovery of the DISC1 (disrupted in Schizophrenia 1) gene, found via analysis of a large Scottish family with a high rate of schizophrenia and psychotic affective disorder. All affected members of the family carry a $(1 ; 11)(\mathrm{q} 42.1 ; \mathrm{q} 14.3)$ translocation; the chromosome 1 translocation break point falls between exon 8 and 9 of DISC1, presumably resulting in loss of DISC1 expression. This finding has launched an entire subfield of schizophrenia genetics and neurobiology, with an emphasis on the role of the DISC1 gene product and its protein interaction partners in neurodevelopment and synaptic function (Chubb et al, 2008).

In the past year, DISC1 has been put into the context of key signal transduction pathways and other genetic findings. In one critical study (Mao et al, 2009), DISC1 was shown to modulate the 'canonical' Wntsignaling pathway. This pathway (Komiya and Habas, 2008) is activated when a member of the Wnt family of secreted glycoproteins binds a member of the Frizzled receptor family along with coreceptors. Pathway activation reduces GSK3 $\beta$ kinase activity, resulting in diminished phosphorylation of $\beta$-catenin. Unphosphorylated $\beta$-catenin accumulates in the cytoplasm and is translocated into the nucleus, where it functions as a transcriptional coactivator. Among other effects, this transcriptional activity can drive neuronal neurogenesis. DISC1 directly interacts with GSK3 $\beta$, inhibiting GSK3 $\beta$ phosphorylation of $\beta$-catenin and thus increasing $\beta$-catenin-induced transcriptional activity. The effect is to mimic Wnt pathway activation. Loss of DISC1 inhibits $\beta$-catenin-induced transcription, providing a potential mechanism by which DISC1 loss of function mutations might exert their effect.

DISC1 has also been linked to pathways involving Neuregulin-1 (NRG1), one of the most robust candidate genes for schizophrenia (Mei and Xiong 2008). Extracellular NRG1, cleaved from Pro-NRG1, interacts with and activates the ErbB family of receptor protein kinases (ErbB2, 3, 4, and EGFR), starting a cascade that activates a number of partially overlapping pathways, including RafMEK-ERK and PI3K-Akt. NRG1 signaling has been implicated in neuronal migration, axon guidance, synapse formation, myelination, and oligodendrocyte development. NRG-1 activated Akt inhibits GSK3 $\beta$, tying NRG1 signaling to Wnt and DISC1 activity. The related effects of DISC1 and NRG1 have been highlighted in a zebrafish model, in which DISC1 loss produced developmental deficits very similar to loss of NRG1 signaling, including failure of normal oligodendrocyte development and near total failure of olig2-positive cerebellar neuron development (Wood et al, 2009). The effect of psychotropic agents on these pathways provides an additional link to major mental illness. For instance, lithium activates Akt and inhibits GSK3 $\beta$, whereas antipsychotic agents, by antagonism of D2 receptors, block the stimulatory effect of dopamine on Akt.

Overall, the convergence of genetic, pharmacological, and neurobiological data have opened the door to multiple novel potential therapeutic targets in the DISC1-Wnt-NRG1 systems, and this neurogenetic approach holds considerable promise for future research. For instance, the recent association of the MHC locus on chromosome $6 \mathrm{p}$ with schizophrenia (e.g., Stefansson et al, 2009) supports the long standing concept that environmental factors such as infection may have a role in schizophrenia, and provides a rationale for models of disease that encompass both genetic and environmental factors. Associations of neurogranin on $11 \mathrm{q} 24.2$ and transcription factor 4 (TCF4) on 18q21.2 with schizophrenia (Stefansson et al, 2009) may lead to new pathways with additional therapeutic targets. Psychiatric research has entered an era in which genetic findings implicate specific signaling pathways, leading to new insights into disease pathogenesis and the development of new approaches to therapeutics.

\section{Russell L Margolis ${ }^{1,2,3}$ and Christopher A Ross ${ }^{1,2,3,4}$}

${ }^{1}$ Division of Neurobiology, Department of Psychiatry, Johns Hopkins University School of Medicine, Baltimore, MD, USA: 
${ }^{2}$ Department of Neurology, Johns Hopkins University School of Medicine, Baltimore, MD, USA;

${ }^{3}$ Program in Cellular and Molecular Medicine, Johns Hopkins University School of Medicine, Baltimore, MD, USA and

${ }^{4}$ Departments of Pharmacology and Neuroscience, Johns Hopkins University School of Medicine, Baltimore, MD, USA

E-mail: rmargoli@jhmi.edu

\section{DISCLOSURE}

Potential conflicts of interest for RLM: Consultant for AstraZeneca, grant support from Medivation, Amarin, Pfizer, and Forest Pharmaceuticals.

No conflicts of interest for CAR.

Chubb JE, Bradshaw NJ, Soares DC Porteous DJ, Millar JK (2008). The DISC locus in psychiatric illness. Mol Psychiatry 13: 36-64.

Komiya Y, Habas R (2008). Wnt signal transduction pathways. Organogen 4: 68-75.

Mao $\mathrm{Y}, \mathrm{Ge} X$, Frank CL, Madison JM, Koehler AN, Doud MK et al. (2009). Disrupted in schizophrenia 1 regulates neuronal progenitor proliferation via modulation of GSK3beta/betacatenin signaling. Cell 136: 1017-1031.

Mei L, Xiong WC (2008). Neuregulin 1 in neural development, synaptic plasticity and schizophrenia. Nat Rev Neurosci 9: 437-452.

Stefansson H, Ophoff RA, Steinberg S, Andreassen OA, Cichon S, Rujescu D et al. (2009). Common variants conferring risk of schizophrenia. Nature 460: 744-7.

Wood JD, Bonath F, Kumar S, Ross CA, Cunliffe VT (2009). Disrupted-in-schizophrenia 1 and neuregulin 1 are required for the specification of oligodendrocytes and neurones in the zebrafish brain. Hum Mol Genet 18: 391-404.

Neuropsychopharmacology Reviews (2010) 35, 350-351; doi: I 0.1038/npp.2009.137

\section{Antidepressants, age, and neuroprogenitors}

New neurons are generated in the granule cell layer of the dentate gyrus (DG) of the hippocampus in adult humans (Eriksson et al, 1998). Our knowledge of adult neurogenesis in humans is quite limited and it could differ from adult neurogenesis in lower mammals. In rodents, neurogenesis is necessary for learning, and some antidepressant effects are lost in the absence of adult neurogenesis, which increases with environmental enrichment and exercise (Olson et al, 2006), as well as with antidepressant treatment (Couillard-Despres et al, 2009).
We reported (Boldrini et al, 2009) that selective serotonin reuptake inhibitors and tricyclic antidepressants increase dividing and neural progenitor cells (NPCs) in the DG of depressed subjects (MDD), compared with untreated MDD patients or controls. In humans, antidepressants increase the number of mitotic cells of all phenotypes in the DG, regardless of age. On the other hand, replication of NPCs, as in lower mammals, decreases with age (Couillard-Despres et al, 2009). This might explain why there is a poor antidepressant response in the elderly.

The functional relevance of the enhancement of neurogenesis by antidepressants needs to be ascertained by determining whether increased cell proliferation is associated with improvement of symptoms in MDD. In our study (Boldrini et al, 2009), a significant proportion of subjects died by suicide, which would argue against the benefits of antidepressant-induced cell proliferation, as opposed to the potential benefits of cell maturation, survival, and integration into functional neural networks, which should have a greater role in the potential beneficial impact of adult neurogenesis. Exposure to enriched environments, learning, and neurotrophins improve the survival and differentiation of newborn cells. Factors regulating cell survival and integration should be considered when examining the role of adult neurogenesis for antidepressant efficacy.

Another open question is the role of neurogenesis in the pathogenesis of MDD. Adult neurogenesis decreases with stress in rodents and is enhanced by environmental enrichment, exercise, and antidepressants. However, blunted cell replication alone does not induce depression-like behavior in mice. Growth factors, which affect neurogenesis, are decreased in MDD. Therefore, impaired hippocampal plasticity may be involved in the pathogenesis of MDD, not merely because of impaired cell replication but also because of impaired cell connectivity and functional inte- gration into brain circuits that regulate emotional responses to the environment.

In our study, the antidepressantinduced increase in NPCs and dividing cells was associated with a larger volume of DG. Antidepressant treatment is known to increase hippocampal volume in posttraumatic stress disorder (Bossini et al, 2007), but no similar data are available in depression, although patients with MDD have a smaller hippocampus. The volume increase could be related to a restoration of cell number or neuropil, as antidepressants reverse dendritic shrinkage and improve cell survival, activating the antiapoptotic protein $\mathrm{Bcl}-2$ and brain-derived neurotrophic factor expression in mammals.

Future studies must determine whether antidepressant response is linked to increased neurogenesis, but assessing adult neurogenesis in vivo is challenging. In a recent study, magnetic resonance spectroscopy was proposed as a possible method, but the specificity of the molecule used to identify NPCs was questioned and the results have not been replicated. Positron emission tomography has the limitation of low resolution and the unknown consequences of radiolabeling newborn cells. Cerebral blood volume, which correlates with angiogenesis, may prove to be a viable method for detecting neurogenesis in vivo (Pereira et al, 2007). Linking neurogenesis to improvement in depression symptomatology would justify seeking new treatments that increase not only neurogenesis but also plasticity, cell survival, and integration into functional networks.

\section{ACKNOWLEDGEMENTS}

This study was supported by PHS Grant nos MH40210, MH62185, MH64168, and MH083862, the American Foundation for Suicide Prevention, the Diane Goldberg Foundation and the Janssen Fellowship in Translational Neuroscience. 